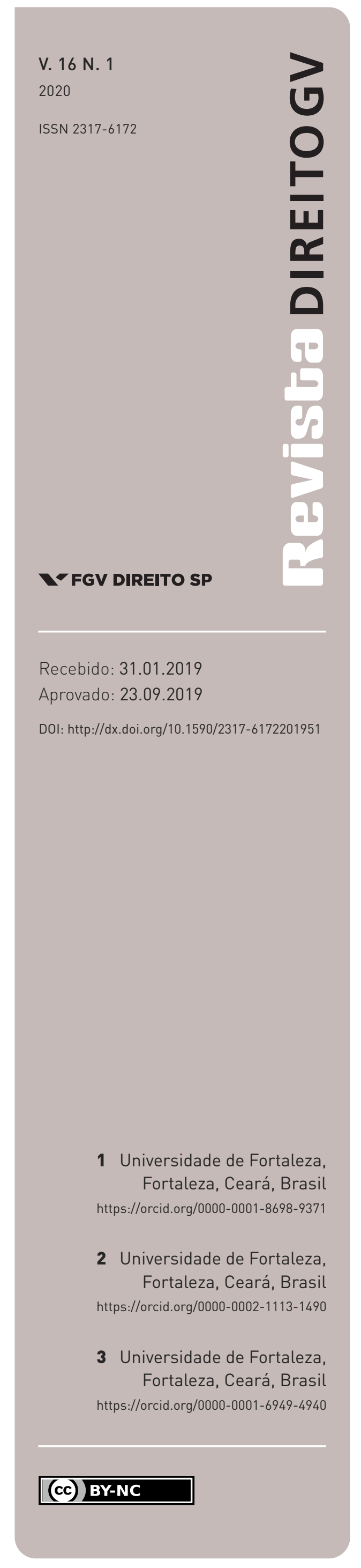

\title{
Legal tech: analytics, inteligência artificial e as novas perspectivas para a prática da advocacia privada
}

LEGAL TECH: ANALYTICS, ARTIFICIAL INTELLIGENCE AND THE NEW PERSPECTIVES FOR THE PRIVATE PRACTICE OF LAW

\author{
Mariana Dionísio de Andrade 1 , Beatriz de Castro Rosa ${ }^{2}$ \\ e Eduardo Régis Girão de Castro Pinto
}

\section{Resumo}

Esta pesquisa tem como objetivo responder ao seguinte questionamento: de que maneira a inteligência artificial e plataforma Litigation Analytics podem contribuir para a prática da advocacia privada? Para responder ao questionamento, é necessário conhecer o impacto do contínuo desenvolvimento da inteligência artificial para a prática jurídica e os benefícios da aplicação das novas tecnologias em um contexto legal tech, o que inclui o uso de ferramentas como o sistema E-SAJ. É essencial que haja complementaridade entre as pesquisas quantitativa e qualitativa, justamente para que seja possível interpretar os dados e replicar os métodos de cada estudo. Para construir a pesquisa, foi utilizada a abordagem teórico-analítica para relacionar a inteligência artificial e os sistemas de automação presentes no cotidiano do Poder Judiciário brasileiro à atuação profissional da advocacia privada. A pesquisa é exploratória, e os procedimentos adotados seguem uma abordagem qualitativa. É possível concluir que mecanismos de inteligência artificial baseados em plataformas analytics tendem a conferir maior previsibilidade para a advocacia privada, porque possibilitam a avaliação sobre as chances aproximadas de recepção das demandas, definição mais adequada de custos envolvidos, melhor desenho de estratégias para a argumentação jurídica e, principalmente, melhor compreensão dos padrões de comportamento presentes nos julgamentos.

\section{Palavras-chave}

Inteligência artificial; legal tech; analytics aplicada à prática jurídica; Sistema de Automação da Justiça (SAJ); Litigation Analytics.

\section{Abstract}

This research aims to answer the following question: how artificial intelligence and Litigation Analytics platform can contribute to the private practice of law? To answer the question, it is necessary to know the impact of the continuous development of artificial intelligence for legal practice and the benefits of applying new technologies in a legal tech context, wich includes mechanisms as the e-SAJ system. It is essential that quantitative research be complementary to the qualitative one precisely so that it is possible to interpret data and replicate the methods of each study. To construct the research, the theoretical-analytical approach was used to relate the artificial intelligence and the automation systems present in the daily life of the Brazilian Judiciary to the professional performance of private practice of law. The research is exploratory and the adopted procedures follow a qualitative approach. It is possible to conclude that artificial intelligence mechanisms based on analytics platforms tend to give greater predictability to private attorney because they allow an evaluation of the approximate chances of receiving the demands, more appropriate definition of costs involved, better design of strategies for juridical argumentation and, especially, a better understanding of the patterns of behavior present in the trials. 


\section{Keywords}

Artificial intelligence; legal tech; analytics applied to legal practice; System SAJ;

Litigation Analytics.

\section{INTRODUÇÃO}

Este artigo pretende responder ao seguinte problema de pesquisa: de que maneira a inteligência artificial e a plataforma Litigation Analytics podem contribuir para a prática da advocacia privada? As profissões jurídicas convivem em um contexto de contínuas inovações tecnológicas, o que exige constante atualização e aprendizado sobre as novas ferramentas, plataformas e softwares disponíveis não apenas para a pesquisa jurídica, mas também para a análise jurídica. O recorte analítico se concentra na apresentação de algumas ferramentas de inteligência artificial, especialmente o Sistema de Automação da Justiça (SAJ), selecionado pela relevância e pioneirismo de suas funcionalidades e pela amplitude de utilização pela advocacia privada.

O texto se divide em três seções. A primeira aborda a inteligência artificial e sua aplicação no cotidiano forense e na prática jurídica, sobretudo no que diz respeito às ferramentas para análise jurídica, introdução e procedimentos do SAJ e à demonstração de dados sobre a virtualização processual e seus efeitos, com base em informações disponibilizadas pelo relatório Justiça em Números, do Conselho Nacional de Justiça (CNJ), de 2018. Além disso, apresenta algumas soluções legal tech, como o Sistema SAJ ADV, o Convex Legal Analytics, o PeticionaMais e o Cofre Virtual, e descreve, ainda, o funcionamento dos sistemas SAJ Ministérios Públicos, SAJ Tribunais e SAJ Procuradorias.

A segunda seção se concentra no uso de tecnologias para pesquisa e análise como um meio útil e inovador para conferir cientificidade à prática jurídica, citando exemplos de softwares que identificam, por análise comparativa de dados, possíveis tendências e inclinações de tribunais e julgadores.

A terceira seção destina-se a enfrentar a pergunta de pesquisa, verificando de que maneira o uso de inteligência artificial pode promover benefícios reais para a advocacia privada, relacionando a plataforma Litigation Analytics como mecanismo hábil à predição de comportamentos dos julgadores na primeira e na segunda instâncias, auxílio na ampliação das técnicas de desenho de estratégias para o litígio e gerenciamento de clientes nos escritórios.

A presente pesquisa foi realizada com base em estudo descritivo-analítico, com aportes teóricos com fundamento, principalmente, em textos de Reichel Beithon, Abdul Paliwala e Mark K. Osbeck, para a construção das ideias de integração metodológica. 
A pesquisa foi desenvolvida com o intuito de obter não apenas o maior aproveitamento quanto ao assunto abordado, mas, principalmente, a produção científica de maior alcance e produtividade para o ambiente acadêmico, especialmente para as ciências jurídicas. O estudo tem relevância teórica porque oferece uma contribuição inédita e original sobre o tema, além da descrição sobre sistemas de automação, na tentativa de propiciar aos profissionais da área uma perspectiva inovadora e concreta sobre a presença das plataformas de inteligência artificial no cotidiano da advocacia privada.

Ainda, possui relevância prática, na medida em que oferece alternativas viáveis, parcimoniosas e pouco onerosas para a análise jurídica, o que tende a fortalecer a atuação profissional, conferir um nível valioso de predição sobre as circunstâncias de cada juízo e, por conseguinte, promover um avanço na relação entre clientes, advogados e Poder Judiciário.

\section{INTELIGÊNCIA ARTIFICIAL APLICADA À PRÁTICA JURÍDICA: FERRAMENTAS PARA LEGAL} ANALYTICS

O cerne da atuação jurídica na advocacia privada consiste na elaboração de teses e argumentações para a defesa ou composição de interesses, a partir da aplicação da norma jurídica ao caso concreto. Para identificar leis relevantes - estatutos, casos, regras ou mesmo precedentes, e aplicá-las aos fatos, advogados têm conduzido pesquisas jurídicas na tentativa de predizer com um mínimo de segurança qual seriam as argumentações jurídicas mais tendentes ao êxito. Assim, é inquestionável que a realização de pesquisas jurídicas precisas continua a ser uma habilidade fundamental de uma advocacia de sucesso, afastando chances de perda e fortalecendo o raciocínio legal.

Para conferir maior cientificidade a essas pesquisas, tão relevantes para a atuação profissional de magistrados, analistas, técnicos e da própria advocacia privada, a utilização adequada de ferramentas consiste em um caminho fundamental. Para otimizar essas pesquisas, a análise jurídica tem se apresentado como alternativa interessante.

Para uma análise jurídica eficiente e baseada em padrões de previsibilidade, a inteligência artificial se apresenta como ferramenta útil na medida em que auxilia na redução de tempo, na otimização de funções e na melhor identificação de termos para consulta, automatizando pesquisas e reduzindo o esforço sobre demandas que poderiam ser programáveis (PERROTA e COSTA FELIPE, 2018).

A pretensão da automação nas buscas não significa, todavia, uma evolução imediata na operacionalização e alimentação dos sistemas. Para que as funcionalidades se realizem de maneira eficiente, a capacidade de disponibilização dos dados deve acompanhar a necessária rapidez das informações. Ainda, mesmo as ferramentas de inteligência artificial precisam de tempo para abranger toda a complexidade das questões jurídicas (OLIVEIRA e COSTA, 2018).

Não se fala aqui em sublimar a função do julgador como hermeneuta e a necessária cautela na exegese sobre os casos peculiares, tampouco na substituição à atividade humana; mas, 
sim, na otimização das funções pelo auxílio de mecanismos econométricos de mensuração e consulta.

A análise jurídica com base em mecanismos econométricos (analytics) envolve dados de mineração contidos em documentos para, em seguida, reunir esses dados para fornecer insights anteriormente desconhecidos sobre o comportamento dos indivíduos (juízes, desembargadores e advogados), organizações (partes, tribunais, escritórios de advocacia) e assuntos de ações judiciais (v.g., patentes ou contratos) que preenchem o sistema de litígios. O sistema analytics pode revelar tendências e padrões em litígios anteriores e informar a estratégia legal, antecipando os resultados nos casos atuais e conferindo certo nível de predição para a atuação dos advogados.

Os insights orientados por dados da análise jurídica (legal analytics) não substituem a pesquisa ou o raciocínio jurídico, tampouco a pesquisa pontual realizada pelos advogados. Contudo, constituem-se em um complemento, antes e durante o litígio (KEISER, 2017).

Entretanto, é necessário esclarecer a distinção fundamental entre pesquisa jurídica e análise jurídica. Enquanto a primeira se destina à busca por decisões jurisprudenciais, julgados, a segunda depende de tecnologias avançadas, como o uso de softwares específicos e processamento de linguagem natural, para limpar, estruturar e analisar metadados e documentos.

Os avanços promovidos pela tecnologia da informação, aliada ao desenvolvimento de softwares de análise de metadados, podem constituir em uma nova era para o Direito, porque tornam possível a superação de desafios como a lentidão para a análise processual, a redução do tempo de discussão sobre incidentes de resolução de demandas repetitivas pela criação de padrões e a baixa qualidade da alimentação dos sistemas pela aplicação de informações coesas e pautadas em uma metodologia uniforme. Podem, ainda, contribuir com a redução dos indicadores de congestionamento e promover um incremento real na produtividade (JUNQUILHO e MAIA FILHO, 2018).

A análise jurídica oferece aos litigantes respostas baseadas em fatos para as principais questões que surgem durante o curso da marcha processual, como: "Quais são as predileções do juiz X?"; "Qual a inclinação para a recepção de determinado recurso em determinada turma do Tribunal de Justiça?”; "Quais táticas específicas são utilizadas por advogados diferentes em casos semelhantes?”; "A parte já protocolizou demandas do mesmo jaez ou com semelhante causa de pedir em momentos anteriores? Se sim, quais foram os resultados?"; "Casos como este são mais tendentes ao consenso ou devem prosseguir no contencioso tradicional?”; “Qual é o histórico de litígios da empresa Z nesse tipo de demanda?”; "Quais empresas têm mais experiência nessa área e quais foram os resultados desses casos?”.

Com o analytics, os litigantes podem responder a essas perguntas com rapidez e precisão, chegando em minutos a respostas abrangentes que levariam semanas ou ainda mais tempo para serem obtidas manualmente, pela busca tradicional em várias fontes. Ferramentas como o Litigation Analytics, presente na plataforma de inteligência artificial Westlaw Edge, auxiliam litigantes a gerenciar melhor as expectativas do cliente, entender o posicionamento 
dos juízes designados, obter informações competitivas sobre os advogados da parte contrária e ter acesso a um suporte de pesquisa jurídica direcionado à formulação de estratégias, uma vez que consegue, com base em decisões anteriores, prever como um juiz pode decidir sobre o caso.

Na plataforma Litigation Analytics, o profissional da advocacia insere os dados em um software que realiza a mineração e verifica a compatibilidade das informações para estabelecer um padrão de forma automatizada. A plataforma realiza a pesquisa entre os documentos a partir da análise de conteúdo (compatibilidade por palavras-chave ou termos relacionados que são anteriormente definidos pelo pesquisador) para localizar argumentos, problemas e padrões de fatos aplicáveis ao caso. Além disso, o Litigation Analytics economiza o tempo de pesquisa em todo um registro, que pode ter centenas de páginas (v.g., julgados completos de uma turma em uma periodização superior a dez anos). A partir da lista de resultados, os pesquisadores passam a ter acesso aos resultados, com base em cálculos com algoritmos que identificam a compatibilidade dos termos de busca. A consulta comporta restrições e refinamento de busca quanto a local, nome do julgador, nome do advogado ou do escritório, tribunal e tipo de caso (BEITHON, 2019).

Ao contrário da pesquisa jurídica, a análise jurídica pode ser usada para encontrar respostas rápidas para questões práticas. Em um tempo significativamente menor, advogados podem obter respostas orientadas por dados para perguntas do cliente que, de outra forma, levariam semanas para serem reunidas.

Embora tenham ocorrido mudanças significativas na aplicação da inteligência artificial no campo da atividade jurídica, elas resultaram principalmente de processos comuns de tecnologia da informação, como processamento de dados, armazenamento, recuperação e gerenciamento de dados, combinados com redes de comunicações (PALIWALA, 2016).

O poder e a velocidade da análise jurídica capacitam os advogados a explorar e experimentar diferentes cenários jurídicos e estratégias para oferecer a melhor e mais bem informada orientação ao jurisdicionado. Em um cenário hipotético, um advogado pode ter uma estratégia específica de litígio em mente, mas o cliente entende que a condução do caso deve seguir em uma direção diferente. Usando a plataforma analytics, o advogado pode realizar uma série de pesquisas e ajustar parâmetros para fornecer ao cliente uma gama de resultados possíveis para diferentes abordagens legais.

Testar uma estratégia contra dados de resultados de casos anteriores semelhantes e explorar alternativas, se as perspectivas para essa estratégia parecerem pouco promissoras, representa o poder e a promessa da análise jurídica. Contudo, essas análises não seriam possíveis sem a introdução de um sistema de automação unificado para o Poder Judiciário brasileiro, como no caso da virtualização dos processos e informatização das dinâmicas.

O desempenho da informatização no sistema de justiça brasileiro tem sido acompanhado pelo CNJ, que, entre outras atribuições, fiscaliza a introdução de sistemas de automação nos tribunais. 
A partir de pesquisas baseadas em confronto de dados e pautadas em bases econométricas, é possível conhecer a dinâmica de alguns tribunais e, consequentemente, estabelecer o cumprimento de metas; perceber quais tribunais cumprem tais metas e delimitar as razões para o eventual descumprimento; verificar o tipo de ação mais recorrente em cada instância, o volume recursal e os tipos de tese de defesa utilizados recorrentemente em casos semelhantes; fortalecer as teses sobre incidentes de demandas repetitivas; reafirmar valores propostos nas legislações específicas por meio da análise de resultados, utilização racional dos dados digitalizados pelos processos eletrônicos (que, por si sós, já criam um amplo banco de dados para consulta e criação de padrões), etc. (ANDRADE, 2018).

\section{I O Sistema de Automação da Justiça (SAJ)}

O Poder Judiciário brasileiro tem se apoiado em uma ferramenta de transformação digital fundamental para a atualização dos processos e realização mais célere de determinados atos processuais. O SAJ consiste em uma solução tecnológica voltada ao gerenciamento de um número considerável de processos em tramitação na Justiça estadual, com o objetivo de promover a integração entre instituições do Poder Judiciário, sujeitos do processo e jurisdicionados.

É possível perceber os efeitos das decisões sobre o jurisdicionado e o impacto no sistema de organização do Poder Judiciário, auxiliando os órgãos de controle na fiscalização e na construção de metas, especialmente no que diz respeito à virtualização de procedimentos, implantação de processos eletrônicos e ampliação da rede de utilização da inteligência artificial (VISSER, 2006).

A inteligência artificial também supõe uma evolução na implantação de processos judiciais eletrônicos, maior observação sobre a necessidade de aproveitamento dos sistemas já adotados e aprimoramento da articulação entre os diversos setores do Poder Judiciário e todos os sujeitos do processo, para que se comuniquem de maneira coerente e para que todos os atores do sistema de justiça exerçam suas atividades de maneira integrada.

A partir dos dados disponibilizados pelo CNJ, é possível perceber o aumento do percentual de processos que ingressam eletronicamente no Poder Judiciário brasileiro, em primeiro e segundo graus, crescendo linearmente, em curva acentuada, desde 2012 (Gráfico 1). Verifica-se também que a virtualização é mais destacada em segundo grau, uma vez que ainda há estados da Federação cujos sistemas de automação carecem de maiores ajustes no primeiro grau. 


\section{Gráfico 1 - PANORAMa da VirtualizaçÃo de PROCESSOS EM PRimeiro E SEGUNDO GRAUS NO PERÍODO DE 2009 A 2017}

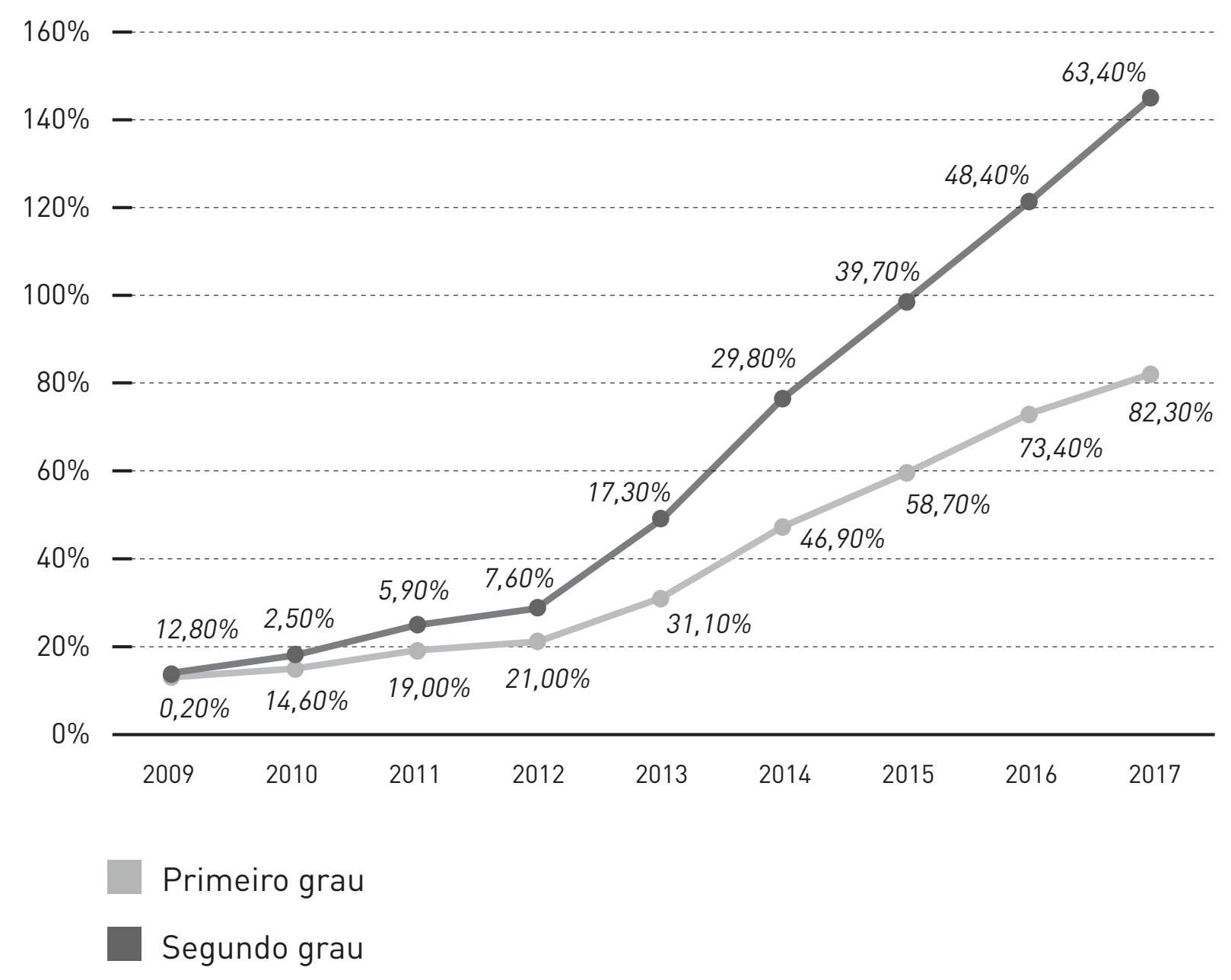

Fonte: Elaboração própria com base nos dados disponibilizados pelo relatório Justiça em Números, do $\mathrm{CNJ}$ (CNJ, 2018).

Segundo dados do relatório Justiça em Números (CNJ, 2018), o índice de novos casos eletrônicos em 2017 tem sido consideravelmente maior em primeiro grau, o que evidencia maior interação entre sistemas em níveis diferentes de jurisdição, conforme descrito pelo Gráfico 2. 
GRÁFICO 2 - ÍNDICE DE NOVOS CASOS ELETRÔNICOS EM PRIMEIRO E SEGUNDO GRAUS, POR TRIBUNAL DE JUSTIÇA, NO PERÍODO DE 2017

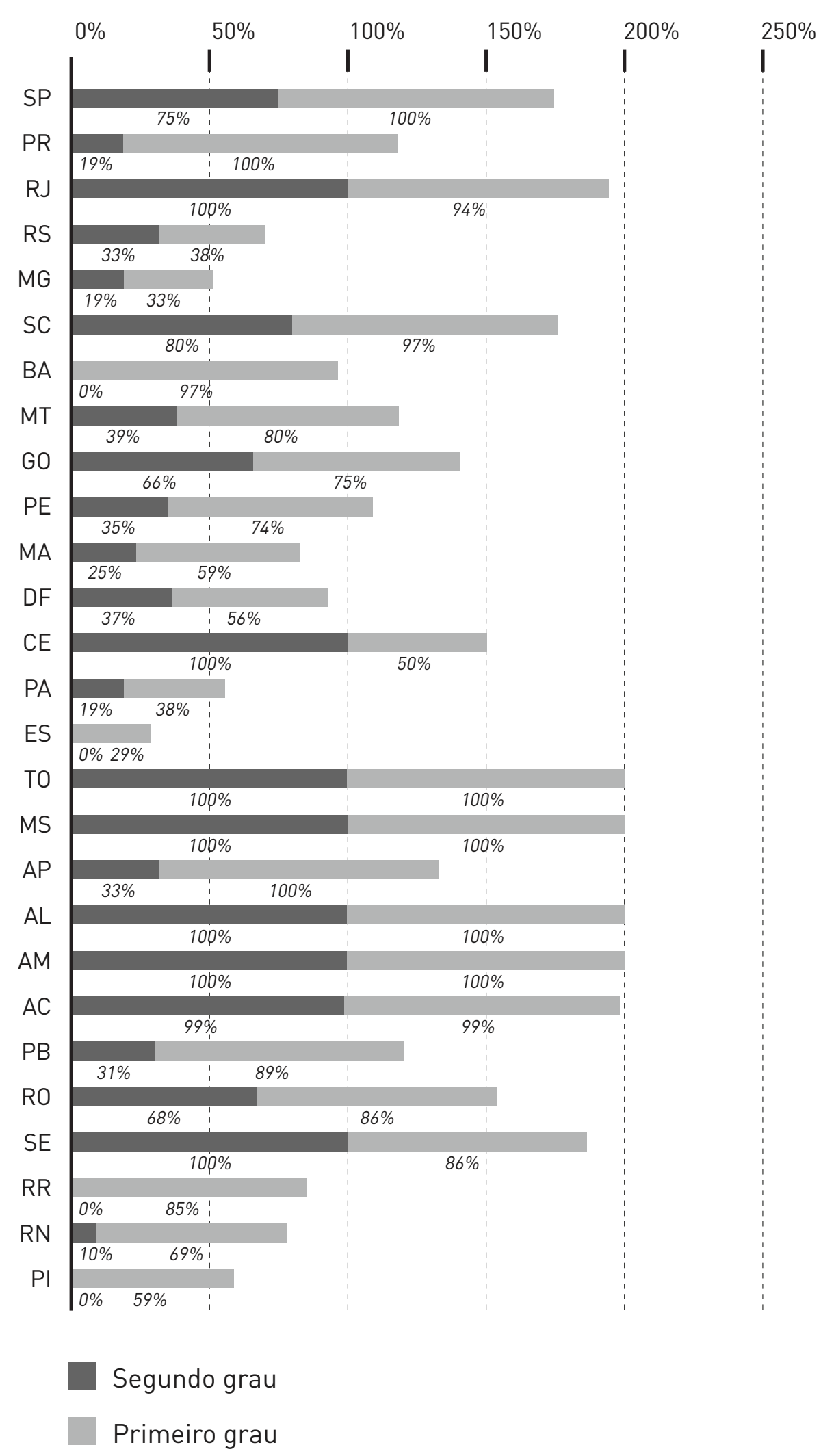

Fonte: Elaboração própria com base nos dados disponibilizados pelo relatório Justiça em Números, do $\mathrm{CNJ}$ (CNJ, 2018).

A Resolução CNJ n. 185, de 2013, que inseriu o Sistema Processo Judicial Eletrônico (PJe) como mecanismo de processamento de informações e prática de atos processuais, influenciou 
de maneira considerável o percentual de processos autuados eletronicamente, que passou de 30,4\%, em 2013, para 79,7\%, em 2017 (CNJ, 2018).

O aprimoramento dos indicadores depende de uma capacitação contínua na utilização de sistemas de automação, conferindo maior precisão na verificação do tempo de tramitação, melhor otimização dos procedimentos e efetivo dinamismo nas diferentes instâncias.

A virtualização de processos, como um indicador exemplificativo da inserção de novas tecnologias de acesso e armazenamento, evidencia avanços tecnológicos absolutamente necessários para a área jurídica, auxiliando a atuação da advocacia privada, sobretudo no que se refere ao envio de petições, de recursos e a prática de atos processuais em geral.

É possível reconhecer os sistemas de automação e virtualização como uma ferramenta blockchain, porque a assinatura digital exige certificação digital emitida por autoridade certificadora e o credenciamento demanda registro para o acesso ao sistema, de modo a preservar o sigilo, a identificação e a autenticidade de suas comunicações, ou mesmo em razão da utilização de tecnologia para o armazenamento e processamento de dados.

A introdução de sistemas de automação consiste em uma inovação bem-recepcionada, não por se tratar da mera adoção de novas tecnologias, mas fundamentalmente por promover uma real modificação no cenário da produtividade, uma vez que veio solucionar problemas graves de padronização, além de auxiliar a advocacia privada com a maior acessibilidade aos processos e maior transparência quanto a diversos atos processuais. Ainda, a utilização dos sistemas de automação permite um gerenciamento centralizado, diminuição de custos, aumento na capacidade do Poder Judiciário em atender maiores e diferentes demandas de armazenamento, processamento e consultas de dados.

A tecnologia aplicada no funcionamento do SAJ, por exemplo, é atualizada constantemente para simplificar tarefas típicas do cotidiano forense, agilizar procedimentos e proporcionar maior dinamismo ao sequenciamento dos atos processuais.

A cronologia de implantação do SAJ se inicia com a criação do Sistema Softplan em 1990, no qual soluções inovadoras foram propostas com o objetivo de uniformizar a coleta e disposição de dados para consulta, com base em analytics, ciência de dados e inteligência artificial (SOFTPLAN, 2019).

Em 1992, o Tribunal de Justiça de Santa Catarina passa a adotar o SAJ (produto da Softplan) como iniciativa pioneira em todo o país, seguido pelo Tribunal de Justiça de São Paulo (TJSP) em 1997 e, apenas em 2001, pelos Tribunais de Justiça do Acre, do Amazonas, do Ceará, de Alagoas e do Mato Grosso do Sul. Em 2007, o SAJ se integra a Ministérios Públicos e Procuradorias, dando início ao peticionamento eletrônico via portal e-SAJ; e, motivado pela publicação da Lei n. 11.419, de 19 de dezembro de 2006, que dispõe sobre a informatização do processo judicial, o TJSP passou a receber apenas processos digitais, tornando-se exemplo para os demais tribunais como referência de processamento e acompanhamento virtual dos atos. Em 2018, o novo e-SAJ é introduzido como plataforma de utilização geral para todo o Poder Judiciário (BALARDIM, 2015). 
Os Tribunais de Justiça de todo país já se consolidaram na utilização de tecnologias para o peticionamento, acompanhamento, arquivamento, tramitação, diligenciamento eletrônico dos atos, armazenamento ou tráfego de documentos e arquivos digitais; entretanto, ainda não se pode afirmar que todos estão em mesmo nível de paridade quanto ao controle de tempo de falha e de uniformização quanto à integralidade dos procedimentos.

O SAJ se divide em esferas de atuação pontuais para garantir o respeito às peculiaridades e à organização interna de cada órgão. O SAJ Tribunais propicia a minoração no tempo de tramitação de processos que já se encontram digitalizados, além de promover maior otimização das tarefas burocráticas, auxiliando a dinâmica das atividades realizadas por servidores e magistrados, em primeiro e segundo graus. Como principais vantagens do sistema SAJTribunais, é possível citar a tramitação processual mais célere, a produção automática de peças e a distribuição automática de juntadas.

O sistema SAJ Ministérios Públicos realiza a articulação da atividade exercida por procuradores, promotores, analistas e demais servidores, permitindo a integração dos procedimentos e propiciando maior fluidez e agilidade aos atos do Ministério Público.

O sistema SAJ Procuradorias, complementarmente, busca a redução no tempo de tramitação e ajuizamento, vez que, integrado ao SAJ Tribunais, realiza o controle e o registro dos prazos processuais e automatiza o sistema de intimações, independentemente de cronogramas e editores de textos.

O SAJ ADV é uma solução tecnológica desenvolvida, especificamente, para auxiliar a advocacia privada, visto que pode ser acessado de qualquer plataforma on-line e é compatível com sistemas operacionais como Microsoft Windows e Mac OS X, pois opera pelo sistema de computação na nuvem (cloud computing). Pelo SAJ ADV é possível realizar o registro e acompanhamento processual, além de controlar atividades de gestão, receitas e despesas, simplificando a atuação de muitos advogados e escritórios. Hoje, de acordo com informações do portal virtual SAJ DIGITAL, o SAJ ADV organiza e otimiza o trabalho de mais de 15 mil advogados em todo o território nacional (BALARDIM, 2015).

\section{I.2 Soluções legal tech: Convex Legal Analytics, Peticionamais, Cofre Virtual}

A introdução da inteligência artificial nas práticas jurídicas resulta de décadas de estudos voltados à otimização do tempo e à uniformização de procedimentos para conferir maior harmonização e integração administrativa no Poder Judiciário brasileiro. Soluções em legal tech são pensadas para o desenvolvimento contínuo das operações jurídicas, desde as mais simples às mais complexas.

Aqui, serão ilustrados alguns exemplos de ferramentas legal tech que tendem a auxiliar a advocacia privada. A Convex Legal Analytics é uma plataforma cujo objetivo consiste em gerar economia e redução de riscos pela antecipação de chances de êxito em cada demanda, e atua a partir da combinação da jurimetria, análises preditivas e inteligência artificial para prever o posicionamento com base na comparação com litígios anteriores. A plataforma promete a 
análise da probabilidade de ganhos, a identificação de tendências de decisões, a simulação de estratégias e alternativas e a projeção de cenários, a partir de uma análise descritiva e diagnóstica pela combinação de algoritmos (CONVEX, 2019).

A plataforma PeticionaMais reúne, por meio de uma combinação de algoritmos, em um único espaço virtual, petições diferentes para diversos tribunais. Essa plataforma não demanda configurações complicadas e adapta automaticamente a peça jurídica, conforme exigência de cada tribunal, e propõe benefícios como o reaproveitamento de dados, monitoramento de rotinas, adaptação automática de peças no tamanho requerido pelo sistema virtual de cada tribunal, além de uma fiscalização inteligente sobre os sistemas de envio, verificando se estão disponíveis no momento do protocolo. Ainda, trata-se de uma plataforma acessível em qualquer dispositivo móvel conectado à internet (PETICIONAMAIS, 2019).

O Cofre Virtual - compatível com sistemas operacionais como Microsoft Windows e Mac OS X, home banking e também com o site da Receita Federal - consiste em uma ferramenta voltada à segurança da informação, que monitora a proteção do certificado digital, o que é fundamental para o exercício da advocacia. Com a preservação tecnológica do certificado digital, contratos e petições podem ser protocolizados em outros computadores sem a necessidade de tolkens, smart cards ou leitores de cartão. O sistema é compatível com o SAJ Tribunais, o SAJ ADV, o SAJ Procuradorias e o SAJ Ministérios Públicos e permite a assinatura de vários documentos conjuntamente.

\section{O USO DETECNOLOGIAS PARA PESQUiSA E ANÁlise: MAIS UMA ALAVANCA DE}

\section{CIENTIFICIDADE PARA A PRÁTICA JURÍDICA}

Tradicionalmente, é possível verificar o predomínio da abordagem qualitativa sobre a quantitativa nos estudos acadêmicos da área jurídica, especialmente pela necessidade de interpretar a realidade e os fenômenos sociais a partir de elementos discursivos cuja defesa, não raro, se funda na argumentação dialética. Entretanto, é necessário repensar esse modelo, adotando, quando possível, configurações numéricas capazes de sustentar e conferir maior acuidade às análises jurídicas.

É necessário refletir sobre a possível integração entre métodos, uma vez que a complementaridade entre as abordagens, chamada de pluralismo metodológico, pode ser viabilizada a partir da mera adoção de ferramentas para testar hipóteses ou evidências empíricas, conferindo maior sustentação às teorias propostas pelo pesquisador. Trata-se de uma preocupação inferencial, absolutamente elementar para o avanço disciplinar (REZENDE, 2014).

A análise de dados não é um método de pesquisa, mas, sim, estratégia que pode ser inserida em quaisquer abordagens, sejam quantitativas ou qualitativas. Nesse sentido, o uso da inteligência artificial aplicada à pesquisa jurídica se apresenta como ferramenta de grande valia.

Não só a prática nos tribunais sofre a influência da presença de novas tecnologias auxiliares ao Direito, mas, em grande medida, a pesquisa jurídica ficou mais inteligente, graças a toda uma nova geração de ferramentas alimentadas por tecnologia artificial e processamento de 
linguagem natural, ficando ainda mais expansiva em razão das ferramentas de pesquisa que consideram o uso de dados para a constatação de fenômenos.

A pesquisa jurídica, tradicionalmente teórica e pautada nas análises de base qualitativa, tem sido fortemente influenciada pelo uso de técnicas econométricas e auxiliada pela análise quantitativa. As plataformas de pesquisa jurídica contam com o crucial apoio de tecnologias capazes de tratar dados e reunir informações confiáveis, sobretudo nas análises que consideram o comportamento de tribunais. Como exemplos, há plataformas como Judicata, Vincent (da vLex), Attorney IO e Case IQ(da Casemine), LexisNexis, Lexis Advance e Lexis Analytics, Fastcase e Casemaker, Analyst Workbench, Litigation Analytics (da Westlaw Edge), Gavelytics, Convex Legal Analytics, entre outras possibilidades de espaços úteis de busca e pesquisa.

Com a utilização de tais ferramentas, a produção científica voltada para a área jurídica tende a se aprimorar, o que é essencial para conferir maior qualidade e atualidade dos textos. Entretanto, para que essa utilização passe a fazer parte do cotidiano dos advogados, reputa-se fundamental e necessária a educação continuada em tecnologia, a atualização em métodos de investigação científica para a atuação profissional e acadêmica e a conscientização coletiva sobre a relevância da inserção de novas tecnologias para o exercício da profissão.

A pesquisa teórica tradicional continua consistindo em um essencial mecanismo de consulta, mas é necessário que a comunidade científica passe a reconhecer a fortaleza das pesquisas data driven, não apenas como métodos complementares, mas como formas de desenvolver um novo tipo de abordagem ao campo da pesquisa jurídica.

A jurimetria, que consiste na aplicação das análises baseadas em Ciência de Dados no ramo do Direito, traduz a aplicação de métodos quantitativos que descendem da análise estatística, uma vez que propicia uma visão probabilística de fenômenos de interação no contexto jurídico. A jurimetria fornece uma perspectiva sistemática dos fatores que influenciam ou que exercem algum papel na tomada de decisões pelo magistrado, na medida em que contribui para definir, com suporte em elementos quantitativos, padrões de comportamento legal (ANDRADE, 2018; HILDEBRANDT, 2018).

Há uma série de consequências interessantes para a pesquisa jurídica e para a aproximação entre as decisões e a realidade dos fatos. Com o auxílio de padrões jurimétricos de investigação, é possível identificar e conferir maior transparência e previsibilidade às decisões jurídicas. A investigação jurisdicional também pode aumentar a legitimidade do sistema jurídico, exercendo um papel de supervisão e monitoramento sobre as partes e procedimentos envolvidos (VISSER, 2006; RAMÍREZ, DÍAZ e FERNÁNDEZ, 2016).

A previsibilidade no meio jurídico se baseia em pesquisas large- $N$, ou seja, em bancos de dados com amostras numericamente expressivas e quantitativamente consideráveis. Essa análise de metadados pode auxiliar a identificação de modelos semelhantes de conduta ou padrões de comportamento dos tribunais.

A partir da jurimetria, torna-se possível estabelecer previsões sobre diversos eventos jurídicos, como a noção sobre o tempo de tramitação dos processos, a propensão para a 
realização de acordo por parte do requerido ou a decisão que tende a ser proferida por determinado magistrado.

Considerando a reunião de técnicas como a volumetria, a jurimetria e a legal analytics, é possível desenhar um cenário bem mais seguro para a criação de expectativas sobre causas em andamento. A jurimetria realiza a análise de um número significativo de dados para que informações estatísticas demonstrem os índices de deferimento e indeferimento de processos a partir do histórico de decisões já proferidas em demandas do mesmo tipo, o que permite ao escritório a criação de estratégias mais assertivas e melhor planejamento quanto aos custos envolvidos.

No contexto das decisões judiciais, há processos com características similares, o que sugere a inegável possibilidade de uma verificação de padrão de comportamento dos tribunais e dos julgadores a partir da verificação de julgados, da análise de conteúdo com a utilização de termos-chave, da diferenciação de teses e argumentações jurídicas recepcionadas com êxito e de indicadores de deferimento e indeferimento para demandas com causas de pedir semelhantes.

\section{ANÁlise de ReSUltados: O USO DE INTELIGÊNCIA ARTIFICIAL PODE PROMOVER BENEFÍCIOS REAIS PARA A ADVOCACIA PRIVADA?}

Inicialmente, é necessário refletir sobre os desafios ou possíveis entraves quanto ao uso de ferramentas de inteligência artificial para a advocacia privada. As funcionalidades e possibilidades oriundas da utilização de mecanismos autômatos podem gerar questionamentos aos usuários não apenas em um momento inicial, razão pela qual é fundamental a atualização para melhor adaptação dos profissionais que lidam com novas tecnologias (MARANHÃO, 2017).

Como desafio, pode-se ressaltar a adaptação entre o raciocínio jurídico, complexo por natureza, e a convivência com técnicas próprias das ferramentas de inteligência artificial. As construções normativas envolvem interpretação terminológica e fática, não apenas a reunião ótima de algoritmos. No entanto, a identificação de padrões pode ser realizada por meio de softwares de reconhecimento, a exemplo do Projeto Victor, adotado em matérias de Repercussão Geral pelo Supremo Tribunal Federal (STF). ${ }^{1}$ O significado dos termos de identificação deve considerar a amplitude de expressões e casos concretos, o que só seria possível, em um tempo reduzido, com o uso de inteligência artificial (JUNQUILHO e MAIA FILHO, 2018).

1 O Projeto Victor consiste em uma ferramenta de inteligência artificial criada com o propósito de rastrear, a partir do reconhecimento de padrões aplicados por machine learning, temas de Repercussão Geral em Recursos Extraordinários. O objetivo se concentra em reduzir o tempo de análise dos ministros do STF e conferir maior qualidade às buscas. A iniciativa resulta de uma parceria entre a Universidade de Brasília e o STF (ANDRADE et al., 2020). 
Outro desafio consiste na operacionalização das novas tecnologias pelos profissionais da área, que precisam de capacitação contínua, sobretudo porque o Direito, tradicionalmente, se baseia na interpretação de situações e encadeamento de atos processuais construídos de maneira menos técnica e mais fenomenológica.

O uso de ferramentas inteligentes deve potencializar as consultas e otimizar o tempo gasto com pesquisas por jurisprudência, identificando com maior agilidade possíveis divergências, inclinações decisórias de tribunais e argumentos compatíveis. No entanto, é preciso compreender que a máquina não evidencia um resultado eficiente se a forma de consulta for deficitária. A busca de termos deve se basear em uma metodologia uniforme, assim como a alimentação dos sistemas, para que os outcomes sejam fiáveis (OLIVEIRA e COSTA, 2018).

As diversas ferramentas de inteligência artificial tendem a promover maior previsibilidade para a advocacia privada, a partir do aprofundamento em um campo de pesquisa ainda pouco explorado no Brasil. Trata-se de uma revolução tecnológica reconhecida pelo CNJ, que anunciou em dezembro de 2018 a criação de um laboratório de inteligência artificial para o Processo Judicial Eletrônico (PJe), com base em uma iniciativa exitosa promovida pelo Tribunal de Justiça de Rondônia, que desenvolveu um sistema para criação de modelos de inteligência artificial na área de processamento de linguagem natural, semelhante às plataformas analytics (CIEGLINSKI, 2018).

O uso da tecnologia analytics faz parte de uma nova realidade para empresas, organizações e Poder Judiciário. Sobre o tema, é importante destacar a pesquisa realizada pela Advanced Analytics Software em 2018, que entrevistou 477 participantes, entre organizações, empresas públicas e privadas da Europa, do Oriente Médio e da África e profissionais de diferentes áreas, acerca da percepção quanto ao uso da plataforma Analytics (ver Tabela 1).

TABELA 1 - INDICADORES PERCENTUAIS DE PERCEPÇÃO QUANTO AOS BENEFÍCIOS ORIUNDOS DA UTILIZAÇÃO DA PLATAFORMA A NALYTICS (PERÍODO DE CONSULTA: 2018)

PERCENTUAL BENEFÍCIOS VERIFICADOS

72\% INSIGHTS RELEVANTES A PARTIR DOS SEUS DADOS E APRIMORAMENTO PARA

ESTRATÉGIA DE NEGÓcIOS

$60 \%$

MAIOR INOVAC̣ÃO COM A ADOÇÃO DE FERRAMENTAS ANALÍTICAS EM SUAS

ESTRATÉGIAS DE NEGÓCIOS

MENOR TEMPO GASTO NA PREPARAÇÃO DAS INFORMAC̣ÕES 


\section{PERCENTUAL BENEFÍCIOS VERIFICADOS}

$46 \% \quad$ TOMADAS DE DECISÕES MAIS INTELIGENTES E CONFIÁVEIS

42\% MENOR TEMPO NA GERAC̦ÃO DE INSIGHTS

$59 \% \quad$ ESTRUTURA DE DADOS INTEGRADA OU CENTRALIZADA

43\% FORNECIMENTO DE MODELAGEM E ALGORITMOS DE INTELIGÊNCIA ARTIFICIAL E MACHINE LEARNING

PERCENTUAL LIMITAC̣ÕES VERIFICADAS

24\% DIFICULDADES PARA A IMPLEMENTAÇÃO DA INFRAESTRUTURA CORRETA PARA A INTELIGÊNCIA ARTIFICIAL

Fonte: Elaboração própria com base nos dados disponibilizados pela pesquisa Here and Now: The Need for an Analytics Platform, realizada pela Advanced Analytics Software (SAS, 2018).

A partir dos dados expostos pela Tabela 1, é possível perceber que os benefícios relatados pela maioria dos entrevistados se concentram na redução de tempo, no aumento da confiança e da previsibilidade e no fornecimento de algoritmos fiáveis, o que supõe uma padronização de procedimentos. A pesquisa foi realizada com base em entrevista pautada em perguntas fechadas, de múltipla escolha, e utilizando como delimitação de consulta as perguntas sobre a plataforma analytics.

A plataforma analytics agrega valor às atualizações de jurisprudência, na medida em que analisa e identifica grandes volumes de casos para serviços de pesquisa de citações e captura de decisões administrativas de conselhos e tribunais. Além disso, é possível identificar as teses jurídicas mais incidentes e seu resultado por turma ou por tribunal.

A utilização de softwares de análise jurídica que usam algoritmos de busca por padrões pode ser extremamente útil, sobretudo em uma área do conhecimento cujas dinâmicas dependem da força argumentativa. A análise jurídica pode avaliar com menor nível de incerteza uma vasta quantidade de informações contidas em julgados, precedentes e jurisprudência dos tribunais, tão pouco explorados sob a perspectiva da análise sistemática (OSBECK, 2015; CARELESS, 2018).

Muitos clientes dependem da notificação antecipada de eventuais mudanças legislativas que afetam seus negócios, sobretudo no direito contratual, empresarial e internacional. Softwares 
de automação podem fornecer acompanhamento e resumos contínuos de novas propostas legislativas, regulamentos, jurisprudência e mudanças administrativas, a partir do rastreamento periódico de bases de dados jurídicas.

Quando o escritório de advocacia é capaz de oferecer experiência em processos e tecnologia para minimizar os custos para seus clientes, aliando a confiabilidade com a perspectiva mais clara sobre a condução dos litígios, torna-se mais competitivo em relação a seus pares, avançando estrategicamente em um mercado tão seleto. Ainda, se propõe como um de seus serviços a análise de contratos e alertas de atualização legislativa e regulatória com base em tecnologias da informação, evidencia atualidade e preparação para as novas dinâmicas (KEISER, 2017).

A análise jurídica confere aos litigantes relativa vantagem sobre os advogados da parte contrária ao fornecer informações baseadas em dados sobre como juízes, desembargadores, advogados e partes se comportam em casos semelhantes e como eles provavelmente se comportarão em futuras demandas de mesmo jaez. Trata-se de uma ferramenta interessante e absolutamente relevante para a construção de estratégias de litígio informadas.

Tradicionalmente, para conhecer o comportamento de advogados, empresas, juízes ou partes específicas, seria necessário que advogados confiassem nos questionamentos feitos aos colegas que vivenciaram um tipo específico de litígio. No entanto, esses dados, além de não serem testados, muitas vezes compõem um tamanho irrelevante para a construção de uma amostra, demonstrando apenas uma realidade parcial, imprecisa.

Além disso, esses prognósticos, embora muitas vezes bem-fundamentados, são frequentemente tendenciosos, tanto pelo desejo dos clientes de ganhar quanto pelas experiências de seus próprios advogados, que podem estar sugestionados pela própria experiência (ALARIE, NIBLETT e YOON, 2018). A utilização de inteligência artificial baseada em combinações algorítmicas propicia uma análise com maior objetividade, uma vez que opiniões são afastadas e substituídas por comparações lógicas, evitando o viés de seleção nos casos verificados pela randomização. Ainda, algoritmos podem gerar previsões replicáveis, o que impõe validade externa aos procedimentos.

Embora a tecnologia não possa prever infalivelmente o resultado de um caso específico, ela pode fornecer insights que aumentam as chances de uma predição precisa, permitindo que os usuários busquem uma estratégia de litígio mais direcionada e com maior probabilidade de sucesso (WALKER-OSBORN, 2018).

Como benefícios sobre o uso de Analytics, é possível destacar, inicialmente, a ampliação das técnicas de desenho de estratégias para o litígio. Criar a melhor abordagem para o litígio envolve a análise de dezenas de variáveis, geralmente em todos os pontos do problema. Independentemente da experiência, cada caso se apresenta como único, exigindo que os advogados identifiquem a estratégia mais adequada. É possível destacar ainda a compreensão quanto aos tribunais, às jurisdições e às propensões dos juízes para decidir. Trata-se de uma vantagem, na medida em que é possível extrair informações confiáveis para mostrar os tipos de tópicos 
vistos por um juiz e com qual frequência, para que os usuários possam formular a estratégia mais forte possível. A plataforma Litigation Analytics inclui resultados de movimento, como taxas de recepção de apelação em uma mesma turma.

Como vantagem, também urge assinalar o gerenciamento das expectativas dos clientes. Um dos maiores desafios vivenciados pela advocacia privada consiste na preocupação com o atendimento ao cliente, fidelização e pronta resposta às demandas apresentadas, de maneira célere, transparente e efetiva. Se os advogados possuem mecanismos capazes de conferir um prenúncio mais próximo à realidade dos acontecimentos, o diálogo com o cliente se torna mais cristalino, sobretudo no que diz respeito ao provável resultado, custos envolvidos e cronograma do caso, na medida em que há uma noção mais clara acerca dos dados sobre como o juiz costuma decidir sobre casos semelhantes.

A demanda dos clientes pela redução das despesas legais acabou por fornecer o impulso necessário para muitos escritórios de advocacia transformarem seu modelo de negócios. Os avanços tecnológicos fornecem as ferramentas necessárias para que escritórios se tornem mais atualizados na progressão técnica da informação jurídica.

Softwares e plataformas inteligentes, que derivam da jurimetria em uma compreensão algorítmica do Direito, estão irreversivelmente presentes no cenário da análise jurídica e tendem a afetar vários aspectos do trabalho dos advogados, incluindo tarefas que historicamente dependiam do esforço humano especializado, como a tentativa de previsão de resultados judiciais. Essas novas ferramentas de inteligência artificial apresentam novos desafios e novas oportunidades para os profissionais do Direito (HILDEBRANDT, 2018).

Um dos desafios pode ser traduzido pela seguinte pergunta: "Como transformar textos em números para realizar uma análise jurimétrica?”. Para converter informações formatadas em textos para um padrão de algoritmos, é necessário compreender que cada palavra pode ser transformada em códigos binários simples em formato de variável dummy (v.g., a palavra “culpado" pode ser transformada no algoritmo "1", e a palavra "inocente" pode ser transformada no algoritmo “0”) (GUILLOU, 2018).

A curto prazo, é possível esperar maior transparência na administração judiciária, maior eficiência da administração de conflitos, mais qualidade do acesso à justiça, concretização de princípios como razoável duração do processo e eficiência, além de novos desafios para a organização tradicional de escritórios de advocacia. Com as novas tecnologias, os advogados terão o poder de trabalhar com mais eficiência, aprofundar e ampliar suas áreas de especialização. A longo prazo, é difícil prever qual será o impacto das ferramentas de inteligência artificial, mas é possível assinalar que, à medida que os advogados as incorporarem em suas práticas e expandirem sua gama de serviços em nome dos clientes, tendem a desenvolver uma nova era na condução das demandas - mais eficiente e transparente (ALARIE, NIBLETT e YOON, 2018). 


\section{CONCLUSÃO}

A partir de todo o exposto, é possível inferir que a inteligência artificial e plataforma Litigation Analytics podem contribuir fortemente para a prática da advocacia privada, na medida em que o uso de novas metodologias de pesquisa e análise jurídica tendem a reduzir incertezas desde o atendimento ao cliente até a finalização da relação jurídico-processual. A utilização complementar de ferramentas tecnológicas e a própria introdução dos sistemas automatizados, como o e-SAJ, representam uma inovação na dinâmica do armazenamento, processamento e registro de dados no âmbito do Poder Judiciário, seguidos pelo contínuo aprimoramento da legal tech, que se desenvolve e sofre constantes atualizações para atender às demandas de profissionais da área.

Essa redução de incertezas aduz a um esforço contínuo para conhecer mais profundamente o perfil de cada tribunal, compreendendo as peculiaridades de cada turma e identificando padrões de comportamento que podem auxiliar, em grande medida, a atuação profissional de advogados, desembargadores, juízes de primeiro grau e demais sujeitos do processo.

A ideia fundamental presente no uso da inteligência artificial consiste em localizar padrões, identificar compatibilidades e definir um caminho mais seguro para juristas e clientes, ampliando a perspectiva de confiança que é fundamental para a atuação profissional.

O uso de plataformas de consulta baseadas em legal tech pode ser útil para conferir uma previsibilidade mínima quanto à inclinação de uma corte ou turma em decisões colegiadas, uma vez que é possível identificar padrões de comportamento a partir da análise estatística das decisões anteriores.

A inteligência artificial tende a ser frutífera para a verificação do tempo médio de tramitação de determinadas causas, auxiliando escritórios e advogados a traçar probabilidades para os clientes. O uso da inteligência artificial, ainda, tende a desvendar a inclinação de julgadores e relatores porque, a partir da análise de conteúdo realizada com o auxílio de softwares como o NVivo e o Litigation Analytics, é possível compreender estrategicamente quais foram as argumentações jurídicas mais exitosas, o que permite o desenho mais real de estratégias para cada caso.

A tecnologia está transformando o setor de serviços jurídicos, e a resistência de alguns profissionais consiste em uma barreira previsível. Entretanto, é necessário recordar que preocupações semelhantes foram expressas quando do início da virtualização nos tribunais e, hoje, trata-se de uma irreversível realidade, repleta de vantagens para a advocacia privada e para as demais profissões jurídicas. Essa transição aumentou a demanda por advogados especializados, promovendo uma revolução na aprendizagem de ferramentas tecnológicas e maior necessidade pelo desenvolvimento de profissionais atentos às atualizações.

Da mesma forma, a análise jurídica não tornará obsoleto o julgamento e a perícia de advogados experientes. No entanto, permitirá que aqueles que o empregam proporcionem uma representação melhor e mais eficaz em termos de custo para seus clientes e maior competitividade entre os escritórios que optarem por manter em seus quadros advogados atualizados e 
dinâmicos. É possível afirmar que os advogados que adotam a análise jurídica tendem a vislumbrar esses benefícios mais cedo, adquirindo uma vantagem competitiva sobre aqueles que não o fazem.

\section{REFERÊNCIAS}

ALARIE, Benjamin; NIBLETT, Anthony; YOON, Albert H. How artificial intelligence will affect the practice of law. University of Toronto Law Journal, v. 68, n. 1, p. 106-124, 2018.

ANDRADE, Mariana Dionísio de. Utilização do sistema R-studio e da jurimetria como ferramentas complementares à pesquisa jurídica. Revista Quaestio Iuris, Rio de Janeiro, v. 11, n. 2, p. 680-692, 2018.

ANDRADE, Mariana Dionísio de; PINTO, Eduardo Régis Girão de Castro; LIMA, Isabela Braga de; GALVÃO, Alex Renan de Sousa. Inteligência artificial para o rastreamento de ações com Repercussão Geral: o Projeto Victor e a realização do princípio da razoável duração do processo. Revista Eletrônica de Direito Processual, Rio de Janeiro, v. 21, n. 1, p. 312-335, 2020.

BALARDIM, Graziela. Projeto 100\% Digital: distribuição de processos digitais supera a de físicos por dois meses seguidos noTJSP. SAJ Digital, 25 set. 2015. Disponível em: http: / /www.sajdigital.com.br/ tribunal-de-justica/projeto-100-digital-tjsp. Acesso em: 23 jan. 2019.

BEITHON, Rachel. Five reasons to use litigation analytics on Westlaw Edge. 2019. Disponível em: https:// legal.thomsonreuters.com/en/insights/articles/5-reasons-to-use-litigation-analytics-on-westlaw-edge. Acesso em: 20 jan. 2019.

CARELESS, James. Legal analytics can help your firm become more competitive. 2018. Disponível em: https:// www.cba.org/Publications-Resources/CBA-Practice-Link/solo/2018/Legal-analytics-can-help-yourfirm-become-more-com. Acesso em: 03 fev. 2020.

CIEGLINSKI, Thaís. CNJ anuncia a criação de laboratório de inteligência artificial para o PJe. Agência de Notícias. 2018. Disponível em: http://www.cnj.jus.br/noticias/cnj/88132-cnj-anuncia-a-criacao-delaboratorio-de-inteligencia-artificial-para-o-pje. Acesso em: 02 jan. 2019.

CONSELHO NACIONAL DE JUSTIÇA (CNJ). Justiça em Números: 2018. Brasília: CNJ, 2018. Disponível em: https: / / www.cnj.jus.br/wp-content/uploads/2011/02/8d9faee7812d35a58cee3d92d2df2f25.pdf. Acesso em: 03 fev. 2020. 
CONVEX. Convex Legal Analytics. 2019. Disponível em: https://www.convex.la/?utm_source=LPInovacao-Softplan\&utm_medium=LP. Acesso em: 20 jan. 2019.

GUILLOU, Pierre. Como treinar um algoritmo de inteligência artificial (IA) na área do direito? 2 maio 2018. Disponível em: https://medium.com/@pierre_guillou/como-treinar-um-algoritmo-de-inteligênciaartificial-ia-na-área-do-direito-8ae229cfdb9b. Acesso em: 18 jan. 2019.

HILDEBRANDT, Mireille. Law as computation in the era of artificial legal intelligence: Speaking law to the power of statistics. University of Toronto Law Journal, v. 68, n. 1, p. 12-35, 2018.

JUNQUILHO, Tainá Aguiar; MAIA FILHO, Mamede Said. Projeto Victor: perspectivas de aplicação da inteligência artificial ao direito. Revista Direito e Garantias Fundamentais, Vitória, v. 19, n. 3, p. 219 238, 2018.

KEISER, Barbie. Artificial Intelligence and the Law. Online Searcher, v. 41, n. 3, p. 16-20, maio/jun. 2017.

MARANHÃO, Juliano. A pesquisa em inteligência artificial e direito no Brasil. Conjur, 9 dez. 2017. Disponível em: https://www.researchgate.net/publication/330321339_Pesquisa_em_inteligencia_ artificial_e_direito_no_Brasil.Acesso em: 03 ago. 2019.

OLIVEIRA, Samuel Rodrigues de; COSTA, Ramon Silva. Pode a máquina julgar? Considerações sobre o uso de inteligência artificial no processo de decisão judicial. Revista de Argumentação e Hermenêutica Jurídica, Porto Alegre, v. 4, n. 2, p. 21-39, 2018.

OSBECK, Mark K. Using Data Analytics Tools to Supplement Traditional Research and Analysis in Forecasting Case Outcomes. University of Michigan Law School Scholarship Repository, 2015. Disponível em: https: / / repository.law.umich.edu/cgi/viewcontent.cgi?referer=https: / / www.google.com.br / \&httpsredir=1\&article=2618\&context=articles. Acesso em: 12 jan. 2019.

PALIWALA, Abdul. Rediscovering artificial intelligence and law: an inadequate jurisprudence? International Review of Law, Computers \&Technology, v. 30, n. 3, p. 107-114, $1^{\circ}$ set. 2016.

PERROTA, Raquel P. Coelho; COSTA FELIPE, Bruno Farage da. Inteligência artificial no Direito: uma realidade a ser desbravada. Revista de Direito, Governança e Novas tecnologias, Salvador, v. 4, n. 1, p. $1-16,2018$.

PETICIONAMAIS. Home Page. 2019. Disponível em: https://peticionamais.com.br/?utm_source=LPInovacao-Softplan\&utm_medium=LP. Acesso em: 20 jan. 2019. 
RAMÍREZ, René Acosta; DÍAZ, Yadirka Verdecia; FERNÁNDEZ, Yarina Amoroso. Jurimetría: una opción para la sociedad. Serie Científica de la Universidad de las Ciencias Informáticas, v. 9, n. 4, p. 1-10, 2016.

REZENDE, Flavio da Cunha. Fronteiras de integração entre métodos quantitativos e qualitativos na ciência política comparada. Revista Teoria e Sociedade, n. 22, p. 40-74, 2014.

SAS. Advanced Analytics Software. How to maximize the impact of your analytics: Research shows the strategic benefits from investments in an analytics platform. 2018. Disponível em: https://www.sas.com/content/ dam/SAS/documents/marketing-whitepapers-ebooks/ebooks/en/here-and-now-the-need-for-ananalytics-platform-110056.pdf. Acesso em: 03 fev. 2020.

SOFTPLAN. A inovação está em nosso DNA. 2019. Disponível em: https: / /www.softplan.com.br/inovacao. Acesso em: 25 jan. 2019.

VISSER, Johanna. Jurimetrics, safety and security. International Review of Law, Computers and Technology, vol. 20, n. 1/2, p. 123-145, 2006.

WALKER-OSBORN, Charlotte. Artificial Intelligence Automation and the Law. ITNOW, v. 60, n. 1, p. 42-43, 2018.

\section{Como CITAR ESTE ARTIGo:}

ANDRADE, Mariana Dionísio de; ROSA, Beatriz de Castro; PINTO, Eduardo Régis Girão de Castro. Legal tech: analytics, inteligência artificial e as novas perspectivas para a prática da advocacia privada. Revista Direito GV, v. 16, n. 1, jan./abr. 2020, e1951. doi: http://dx.doi.org/10.1590/23176172201951.

\section{Mariana Dionísio de Andrade}

Doutora em Ciência Política pela Universidade Federal de Pernambuco (UFPE). Mestre em Direito Constitucional pela Universidade de Fortaleza (UNIFOR). EspeCIALISTA EM DiREITO Processual Civil pela Unifor. Professora do Curso de Pós- Graduação lato sensu em Direito e Processo Constitucionais e da disciplina Teoria Geral do Processo Civil no Curso de GraduAÇÃO EM DiREITO NA UNIFOR. FormAÇÃO EM LEADERSHIP and Conflict Management PELA Stanford University.

Formação EM MÉTOdOS QuANTITATIVOS PELA UNIVERSIDADE Estadual do Rio de Janeiro (UERJ). Pesquisadora do Grupo Epistemologia e Método na Ciência Política Comparada (CNPQ/UFPE). CoORdenadora do Projeto Pesquisa EMPÍRICA EM DIREITO - PROPED (CNPQ/UNIFOR).

Professora do Curso de Pós-Graduação em Direito Processual Civil da Escola Superior da Magistratura do Ceará (ESMEC/CE). Advogada. mariana.dionisiolaunifor.br 


\section{Beatriz de Castro Rosa}

Doutora em DiReito Constitucional PELA Universidade dE Fortaleza (UNIFOR). Mestre em Direito Constitucional PELA UNIFOR. Professora dos Cursos de Pós-Graduação lato SENSU EM RESPONSABILIDADE CiVIL E DiREITO DO CONSUMIDOR,

DiReito de Família e Sucessões, Mediação e Gestão de Conflitos na UNIFOR. Professora do Curso de Graduação em Direito da UNIFOR. Chefe da Divisão de Pós-Graduação Lato Sensu e Educação Continuada. Advogada.

beatriz_rosalaunifor.br

\section{Eduardo Régis Girão de Castro Pinto}

Doutorando em Direito Constitucional e Mestre em Direito Constitucional PELA Universidade de Fortaleza (UNIFOR).

Professor do CuRso de Pós-Graduação lato SENSU EM

Processo Civil da Escola Superior da Magistratura do Estado do Ceará (ESMeC). Professor dos Cursos de Pós- Graduação lato sensu em Direito Empresarial, Direito dos Contratos e Responsabilidade Civil e Direito do Consumidor, BEM COMO dO CURSO dE GRAduAÇÃO EM DiREITO dA UNIFOR. Coordenador do Projeto Pesquisa Empírica em Direito PROPED (UNIFOR). ASSESSOR JURÍDICO DA CORREGEDORIA- geral do Tribunal de Justiça do Estado do Ceará. Professor do Curso de Pós-Graduação em Direito Processual Civil da ESMEC. eduardogiraolagmail.com 